\title{
Procedimientos propuestos para la evaluación de la deficiencia en enfermedades respiratorias ocupacionales
}

\author{
Juan Carlos Vázquez-García, ${ }^{*} \bowtie$ Francisco Tornero-Applebaum, ${ }^{*}$ Rodrigo Toral-Villanueva ${ }^{\S}$ \\ Rogelio Pérez-Padilla*
}

\begin{abstract}
*Instituto Nacional de Enfermedades Respiratorias Ismael Cosío Villegas, Ciudad de México; *Dirección General de Seguridad y Salud en el Trabajo, Secretaría del Trabajo y Previsión Social; ${ }^{\circledR}$ División de Discapacidad para el Trabajo, Coordinación de Salud en el Trabajo, Instituto Mexicano del Seguro Social.

Trabajo recibido: 08-III-2017; aceptado: 27-III-2017
\end{abstract}

\begin{abstract}
RESUMEN. En México, la protección social de las enfermedades de origen ocupacional está legalmente establecida en la Ley Federal del Trabajo. El mayor número de padecimientos reconocidos por especialidad está compuesto por 47 enfermedades respiratorias de origen ocupacional (ERO). Sin embargo, la tabla de enfermedades de origen ocupacional no había sido actualizada desde 1970. Recientemente, la Secretaría del Trabajo y Previsión Social integró grupos de trabajo con la finalidad de revisar y actualizar la tabla de enfermedades ocupacionales. Un objetivo adicional fue proveer la metodología más apropiada para la evaluación de la deficiencia funcional de los trabajadores que padezcan alguna enfermedad ocupacional. En este artículo se describen en detalle las pruebas de función respiratoria que incluyen el proceso de valuación de las ERO; se revisa su fundamento fisiológico, técnico y clínico, así como su metodología, su proceso de interpretación y las propuestas de valuación del daño funcional, las cuales son congruentes con los criterios internacionales y se centran primordialmente en parámetros objetivos. Finalmente, se hacen las propuestas que a juicio de los autores deben implementarse en mediano plazo en las instituciones encargadas de la evaluación y seguimiento de estas enfermedades.
\end{abstract}

Palabras clave: Enfermedad pulmonar ocupacional, deficiencia respiratoria, pruebas de función respiratoria, espirometría.

ABSTRACT. Social protection for occupational disease is established by the Federal Labor Law in Mexico. Occupational lung diseases are the greatest number of diseases by specialty, including 47 diseases legally protected. However, this law has not been reviewed since the year 1970. Recently, the Secretariat of Labor and Social Welfare of Mexico assembled working groups in order to update the occupational disease protected by law. An additional aim was to provide the methodology and the recommended procedures for evaluating physical impairment in workers. We describe the pulmonary function tests included in the new proposal for assessing respiratory impairment. Their physiological, technical and clinical bases are reviewed, including the interpretation process. Specific recommendations for the practical implementation of this new methodology in the medium term in Mexico are analyzed by the authors.

Key words: Occupational lung diseases, respiratory impairment, pulmonary function testing, spirometry.

\section{ANTECEDENTES}

La protección social de las enfermedades de origen ocupacional fue legalmente establecida en México con la promulgación de la Ley Federal del Trabajo (LFT) en 1936. En esta ley el artículo 326 reconoció 40 tipos de enfermedades profesionales, de las cuales 17 eran infecciosas y parasitarias, 3 de la vista y el oído y 20 correspondían a otras afecciones. En 1956, se adicionaron 9 fracciones más, es decir, nuevas enfermedades. En la reforma de la LFT de $1970,{ }^{1}$ el artículo 513 retoma la tabla previa de enfermedades del trabajo, pero agrega otros 112 padecimientos para contabilizar un total de 161. El mayor número estaba compuesto por 47 enfermedades respiratorias ocupacionales (ERO) que incluyeron 30 padecimientos definidos como neumoconiosis o padecimientos broncopulmonares secundarios a inhalación de polvos o humos de origen mineral, vegetal o animal; además de otras 17 enfermedades de las vías respiratorias relacionadas con la inhalación de gases y vapores.

El artículo 515 de la LFT, el cual no sufrió modificación en la reforma 2012, encomienda a la Secretaría del Trabajo y Previsión Social (STPS) a realizar las investigaciones y estudios necesarios a fin de que el Presidente de la República pueda iniciar ante el poder 
legislativo la adecuación periódica de las tablas de enfermedades de trabajo y de evaluación de incapacidades permanentes de acuerdo al progreso de la medicina del trabajo. Bajo este contexto, la STPS integró grupos de trabajo que incluyeron especialistas clínicos, especialistas en salud en el trabajo, investigadores, académicos y peritos médicos dictaminadores, con la finalidad de actualizar la tabla de enfermedades de trabajo. Además, este proceso de actualización incluyó: 1) determinar de manera precisa a los trabajadores expuestos de acuerdo al tipo de actividad; 2) definir el tipo de daño a la salud de acuerdo al tipo de exposición; 3) actualizar los elementos clínicos, de laboratorio y de gabinete para la adecuada evaluación y diagnóstico de cada padecimiento; y 4) proveer la metodología más apropiada para la evaluación de la deficiencia o de incapacidad permanente de los trabajadores, así como la determinación de su capacidad residual en función a su calidad de vida y aptitud para el trabajo.

El grupo de trabajo de enfermedades del sistema respiratorio propuso para la actualización de la tabla de enfermedades de trabajo (artículo 513) un nuevo proceso de evaluación de la deficiencia respiratoria de acuerdo con las recomendaciones internacionales. Este proceso se basa en una evaluación objetiva sustentada en las pruebas de función respiratoria (PFR), las cuales deben realizarse bajo estándares vigentes internacionales. El objetivo de esta revisión es describir en detalle las PFR que se incluyen en este proceso, su fundamento fisiológico, técnico y clínico, su metodología y proceso de interpretación, así como las propuestas de evaluación de la deficiencia que son congruentes con los criterios internacionales. Finalmente, se hacen las propuestas que a juicio de los autores se deben implementar en el mediano plazo en las instituciones encargadas de la evaluación y seguimiento de estos enfermos.

\section{Enfermedades respiratorias de origen ocupacional}

Las ERO son un grupo de enfermedades crónicas cuya causalidad está ligada a la exposición crónica de contaminantes aéreos directamente relacionados al ambiente de trabajo. ${ }^{2}$ Estas enfermedades contribuyen significativamente a la carga de enfermedad de la población en general, en particular de la población económicamente activa. Se conoce que las enfermedades pulmonares crónicas como el asma, la enfermedad pulmonar obstructiva crónica (EPOC), las enfermedades pulmonares intersticiales y el cáncer pulmonar pueden tener como origen las exposiciones nocivas del ambiente laboral; incluso pueden ser clínicamente indistinguibles en su forma ocupacional y no ocupacional. ${ }^{2}$ Recién, se ha estimado que los agentes del ambiente de trabajo contribuyen etiológicamente entre 1 y $40 \%$ al cáncer de pulmón, entre 10 y $20 \%$ al asma y alrededor del $15 \%$ a la EPOC. ${ }^{2,3}$ Las ERO impactan la calidad de vida y el desempeño laboral de las personas que las padecen, lo cual también depende de la gravedad de las mismas y la frecuencia con que se puedan exacerbar. Además, las exacerbaciones también pueden depender de las exposiciones ambientales en el trabajo. Todo médico, no sólo debe estar familiarizado con la forma de explorar la exposición ambiental y laboral, sino también con los principales aspectos de diagnóstico y manejo de las ERO, además, debe ser un promotor de un ambiente de trabajo sano y seguro.

Las ERO pueden clasificarse de diferentes formas. Sin embargo, para fines clínicos es importante reconocerlas por su presentación clínica, su daño histopatológico primario y los agentes causales a los que se asocian. En las tablas 1 a 4 se resumen las ERO reconocidas por la ley vigente, éstas son agrupadas en enfermedades de la vía aérea por partículas orgánicas, neumonitis por hipersensibilidad y bronquiolitis obliterante y neumonía organizada (tabla 1), neumoconiosis (tabla 2), bronquitis y/o neumonitis agudas o crónicas de origen químico o tóxico (tabla 3) y asfixias por inhalación de gases, humos y vapores (tabla 4). Se especifica el tipo de exposición y su agrupación actual de acuerdo con la nueva propuesta de la STPS. En esta propuesta, las ERO de tipo infeccioso o neoplásicas se encuentran en otros apartados, por lo que no se incluyen en esta descripción.

\section{Deficiencia y discapacidad}

Los términos deficiencia (impairment) y discapacidad (disability) han sido claramente definidos por la Organización Mundial de la Salud. ${ }^{4,5}$ Deficiencia es la anormalidad o pérdida de una estructura corporal o de una función fisiológica. Esta alteración debe ser medida objetivamente, puede clasificarse como temporal o permanente y debe ser graduada en gravedad. Discapacidad se refiere a la restricción o pérdida de la capacidad para realizar una actividad en forma o dentro del contexto considerado como normal para una persona. Este término incorpora el efecto total de la deficiencia sobre la vida del paciente y se afecta por variables como la edad, género, nivel educativo y socioeconómico, medio ambiente, ocupación y requerimientos de energía que pueden limitar a realizar cierto tipo de trabajos. En consecuencia, dos personas con la misma deficiencia (por ejemplo, daño funcional) pueden tener diferente discapacidad, al grado que uno puede estar incapacitado para su empleo y el otro no. La evaluación de la deficiencia es responsabilidad de un 
Tabla 1. Enfermedades de la vía aérea y neumonitis por hipersensibilidad de origen ocupacional.

\begin{tabular}{|c|c|c|c|c|}
\hline \multirow[b]{2}{*}{ Tipo de enfermedad } & \multirow[b]{2}{*}{$\begin{array}{l}\text { Exposición a } \\
\text { polvos y fibras }\end{array}$} & \multicolumn{2}{|r|}{ Tabla vigente (1970) } & \multirow[b]{2}{*}{ Propuesta 2012} \\
\hline & & $\begin{array}{l}\text { Fracción } \\
\text { legal }\end{array}$ & Enfermedad & \\
\hline \multirow{10}{*}{$\begin{array}{l}\text { Enfermedades de la } \\
\text { vía aérea }\end{array}$} & Lana & 1 & Bronquitis por lana & \multirow{8}{*}{$\begin{array}{l}\text { Enfermedad broncopulmonar } \\
\text { por inhalación de polvos o } \\
\text { fibras de origen orgánico } \\
\text { (bronquitis ocupacional) }\end{array}$} \\
\hline & $\begin{array}{l}\text { Pluma, cuerno, hueso, } \\
\text { crin, pelo y seda }\end{array}$ & 2 & $\begin{array}{l}\text { Bronquitis por polvos de pluma, } \\
\text { cuerno, hueso, crin, pelo y seda }\end{array}$ & \\
\hline & Madera & 3 & Bronquitis por polvos de madera & \\
\hline & Tabaco & 4 & Tabacosis & \\
\hline & $\begin{array}{l}\text { Cereales, harina, heno, } \\
\text { paja, ixtle y henequén }\end{array}$ & 7 & $\begin{array}{l}\text { Bronquitis por harinas, cereales, } \\
\text { heno, paja, ixtle y henequén }\end{array}$ & \\
\hline & Algodón & 8 & Bisinosis & \\
\hline & Cáñamo & 9 & Canabiosis & \\
\hline & Lino & 10 & Linosis & \\
\hline & $\begin{array}{c}\text { Inhalación de } \\
\text { sustancias de alto y bajo } \\
\text { peso molecular }\end{array}$ & 11,47 & Asma ocupacional & $\begin{array}{c}\text { Asma ocupacional y síndrome } \\
\text { disfunción reactiva de la vía } \\
\text { aérea }\end{array}$ \\
\hline & $\begin{array}{l}\text { Exposición crónica a } \\
\text { polvos y humos }\end{array}$ & & & $\begin{array}{l}\text { Enfermedad pulmonar } \\
\text { obstructiva crónica }\end{array}$ \\
\hline \multirow{2}{*}{$\begin{array}{c}\text { Neumonitis por } \\
\text { hipersensibilidad }\end{array}$} & Bagazo & 5 & Bagazosis & \multirow{2}{*}{$\begin{array}{c}\text { Neumonitis por } \\
\text { hipersensibilidad }\end{array}$} \\
\hline & Corcho & 6 & Suberosis & \\
\hline $\begin{array}{c}\text { Bronquiolitis } \\
\text { obliterante y } \\
\text { neumonía organizada }\end{array}$ & $\begin{array}{l}\text { Saborizantes artificiales } \\
\text { (diacetilo) }\end{array}$ & & & $\begin{array}{l}\text { Bronquiolitis obliterante y } \\
\text { neumonía organizada }\end{array}$ \\
\hline
\end{tabular}

Tabla 2. Enfermedades pulmonares ocupacionales del tipo de neumoconiosis.

\begin{tabular}{|c|c|c|c|c|}
\hline \multirow[b]{2}{*}{ Tipo de enfermedad } & \multirow[b]{2}{*}{ Exposición a polvos de: } & \multicolumn{2}{|r|}{ Tabla vigente (1970) } & \multirow[b]{2}{*}{ Propuesta 2012} \\
\hline & & $\begin{array}{l}\text { Fracción } \\
\text { legal }\end{array}$ & Enfermedad & \\
\hline \multirow{18}{*}{ Neumoconiosis } & Carbón mineral & 12 & Antracosis & $\begin{array}{c}\text { Neumoconiosis } \\
\text { de los mineros del carbón }\end{array}$ \\
\hline & Silicatos & 17,30 & Silicatosis & \multirow{4}{*}{ Silicosis y silicatosis } \\
\hline & Sílice & 19 & Silicosis & \\
\hline & Talco o esteatita & 27 & Talcosis o esteatosis & \\
\hline & Mica & 29 & Neumoconiosis por mica & \\
\hline & Asbestos & 20 & Asbestosis o amiantosis & Asbestosis o amiantosis \\
\hline & Hierro & 13 & Siderosis & \multirow{11}{*}{$\begin{array}{l}\text { Neumoconiosis debido a otros } \\
\text { polvos inorgánicos metálicos }\end{array}$} \\
\hline & Calcio & 14 & Calcicosis & \\
\hline & Bario & 15 & Baritosis & \\
\hline & Estaño & 16 & Estanosis & \\
\hline & Berilio o glucino & 21 & Berilosis o glucinosis & \\
\hline & Cadmio & 22 & Neumoconiosis por cadmio & \\
\hline & Vanadio & 23 & Neumoconiosis por vanadio & \\
\hline & Uranio & 24 & Neumoconiosis por uranio & \\
\hline & Manganeso & 25 & Neumoconiosis por manganeso & \\
\hline & Cobalto & 26 & Neumoconiosis por cobalto & \\
\hline & Aluminio y bauxita & 28 & Aluminosis & \\
\hline & Abrasivos sintéticos & 18 & Neumoconiosis por abrasivos & $\begin{array}{l}\text { Neumoconiosis debido a otros } \\
\text { polvos inorgánicos no metálicos }\end{array}$ \\
\hline
\end{tabular}


especialista experto, quien debe cuantificarlo, mientras que la determinación de discapacidad es una decisión también experta, pero que requiere considerar variables médicas y no médicas (individuales, familiares, sociales y laborales).

\section{Evaluación de la deficiencia: valoración funcional respiratoria}

Dada la posibilidad de una ganancia secundaria por el dictamen de incapacidad, es esencial que la valoración

Tabla 3. Bronquitis y neumonitis química de origen ocupacional.

\begin{tabular}{|c|c|c|c|c|}
\hline \multirow[b]{2}{*}{ Tipo de enfermedad } & \multirow{2}{*}{$\begin{array}{l}\text { Inhalación de gases, } \\
\text { vapores, humos, polvos } \\
\text { de sustancias químicas }\end{array}$} & \multicolumn{2}{|r|}{ Tabla vigente (1970) } & \multirow[b]{2}{*}{ Propuesta 2012} \\
\hline & & $\begin{array}{l}\text { Fracción } \\
\text { legal }\end{array}$ & Enfermedad & \\
\hline \multirow{13}{*}{$\begin{array}{c}\text { Bronquitis y/o } \\
\text { neumonitis químicas } \\
\text { o tóxicas }\end{array}$} & Ázoe o nitrógeno & 31,41 & Bronquitis por ázoe & \multirow{7}{*}{$\begin{array}{l}\text { Bronquitis o neumonitis debidas } \\
\text { - a sustancias químicas, como } \\
\text { - amoníaco, anhídrido sulfuroso, } \\
\text { ázoe, vapores nitrosos, níquel, } \\
\text { - cloro, azufre, flúor, fosgeno y } \\
\text { cloruro de carbonilo }\end{array}$} \\
\hline & Amoníaco & 35 & Bronquitis por amoníaco & \\
\hline & $\begin{array}{l}\text { Anhídrido sulfuroso } \\
\text { o azufre }\end{array}$ & 36,42 & $\begin{array}{l}\text { Bronquitis por azufre o } \\
\text { anhídrido sulfuroso }\end{array}$ & \\
\hline & Cloro & 39 & Bronquitis por cloro & \\
\hline & $\begin{array}{l}\text { Fosgeno o cloruro de } \\
\text { carbonilo }\end{array}$ & 40 & $\begin{array}{l}\text { Bronquitis por fosgeno o } \\
\text { cloruro de carbonilo }\end{array}$ & \\
\hline & Flúor & 45 & Bronquitis por flúor & \\
\hline & Níquel & & & \\
\hline & Aldehídos & 38 & Bronquitis por aldehído & \multirow{6}{*}{$\begin{array}{l}\text { Bronquitis o neumonitis debidas } \\
\text { - a sustancias químicas, como } \\
\text { - ácido acético, aldehídos, an- } \\
\text { - timonio, éteres clorometílicos, } \\
\text { isocianatos, mercurio y } \\
\text { sulfato de metilo }\end{array}$} \\
\hline & Sulfato de metilo & 46 & Bronquitis por sulfato de metilo & \\
\hline & Antimonio & & & \\
\hline & Éteres clorometílicos & & & \\
\hline & Isocianatos & & & \\
\hline & Mercurio & & & \\
\hline \multirow{11}{*}{$\begin{array}{c}\text { Bronquitis y/o } \\
\text { neumonitis químicas } \\
\text { o tóxicas }\end{array}$} & Formaldehído y formol & 37 & $\begin{array}{l}\text { Bronquitis por formaldehído } \\
\text { y formol }\end{array}$ & \multirow{6}{*}{$\begin{array}{l}\text { Bronquitis o neumonitis debidas } \\
\text { a sustancias químicas como } \\
\text { - ácidos anhídridos, ácido hidro- } \\
\text { brómico, acetileno, bromuro } \\
\text { de hidrógeno, formaldehído, } \\
\text { formol, metilbromuro y ozono }\end{array}$} \\
\hline & Ozono & 43 & Bronquitis por ozono & \\
\hline & Bromuro de hidrógeno & 44 & Bronquitis por bromo & \\
\hline & Ácido hidrobrómico & & & \\
\hline & Acetileno & & & \\
\hline & Metilbromuro & & & \\
\hline & Ácido crómico & & & \multirow{5}{*}{$\begin{array}{l}\text { Bronquitis o neumonitis debidas } \\
\text { a sustancias químicas, como } \\
\text { ácido crómico, boranos, } \\
\text { cloroacetofenona, cobalto } \\
\text { e hidruro de litio }\end{array}$} \\
\hline & Boranos & & & \\
\hline & Cloroacetofenona & & & \\
\hline & Cobalto & & & \\
\hline & Hidruro de litio & & & \\
\hline
\end{tabular}

Tabla 4. Asfixia por gases, humos y vapores químicos.

\begin{tabular}{|c|c|c|c|c|}
\hline \multirow[b]{2}{*}{ Tipo de enfermedad } & \multirow{2}{*}{$\begin{array}{l}\text { Inhalación de gases, } \\
\text { humos y vapores de } \\
\text { sustancias químicas }\end{array}$} & \multicolumn{2}{|c|}{ Tabla vigente (1970) } & \multirow[b]{2}{*}{ Propuesta 2012} \\
\hline & & $\begin{array}{l}\text { Fracción } \\
\text { legal }\end{array}$ & Enfermedad & \\
\hline \multirow{7}{*}{ Asfixias } & Ázoe o nitrógeno & 31 & Asfixia por nitrógeno & \multirow{7}{*}{$\begin{array}{c}\text { Asfixias por gases, humos y } \\
\text { vapores de sustancias } \\
\text { químicas }\end{array}$} \\
\hline & Bióxido de carbono & 32 & Asfixia por bióxido de carbono & \\
\hline & $\begin{array}{l}\text { Butano, etano, metano } \\
\text { y propano }\end{array}$ & 33 & $\begin{array}{l}\text { Asfixia por etano, metano y } \\
\text { propano }\end{array}$ & \\
\hline & Acetileno & 34 & Asfixia por acetileno & \\
\hline & Cianuro de hidrógeno & & & \\
\hline & Monóxido de carbono & & & \\
\hline & Sulfuro de hidrógeno & & & \\
\hline
\end{tabular}


se centre en pruebas objetivas, en este caso en PFR. El estudio de la función respiratoria es muy antiguo: los primeros intentos por medir los volúmenes pulmonares se originaron en el siglo XVII y para el año 1800 ya se habían hecho las primeras mediciones de volumen pulmonar por medio de dilución de gases. No obstante, fue hasta 1846 cuando el trabajo realizado por el médico inglés John Hutchinson incorporó a la espirometría como una prueba de uso clínico común para la evaluación de salud y de enfermedad respiratoria. ${ }^{6}$

Con frecuencia se piensa en la respiración solamente como el movimiento de entrada y salida del aire desde y hacia los pulmones. Este desplazamiento de aire se denomina mejor en fisiología como ventilación pulmonar. El proceso respiratorio, además de la ventilación, consiste en la transferencia de gases a nivel alvéolo-capilar, el transporte de oxígeno $\left(\mathrm{O}_{2}\right)$ y bióxido de carbono $\left(\mathrm{CO}_{2}\right)$ en la sangre, una segunda transferencia gaseosa a nivel celular y finalmente la respiración química en la mitocondria donde se incorpora el $\mathrm{O}_{2}$ al ciclo de Krebs para la producción de energía en forma de ATP. ${ }^{7-9}$ El compromiso respiratorio por enfermedad puede ser causante de deficiencia funcional temporal o permanente.

En la actualidad, la evaluación mínima de la función respiratoria requiere considerar la parte mecánica, la de intercambio de gases y la evaluación durante el ejercicio. La parte mecánica se refiere a la resistencia al paso del aire por la vía aérea, al volumen pulmonar y a la rigidez del pulmón y del aparato respiratorio. Varias enfermedades afectan de manera inicial la capacidad pulmonar para el intercambio gaseoso teniendo todavía pruebas normales de mecánica, como las que afectan la circulación pulmonar, las enfermedades intersticiales del pulmón y el enfisema pulmonar. De suerte que puede no haber una buena correlación entre la alteración mecánica y el daño al intercambio gaseoso. También cuando la lesión es inicial puede manifestarse solamente como una demanda adicional como la que se observa en el ejercicio.

Las PFR son una medición extraordinariamente objetiva y cuantitativa de la función pulmonar y, por lo tanto, también son útiles para la medición precisa de la mejoría o el agravamiento de las enfermedades. La espirometría es la prueba más simple, accesible y confiable para evaluar la función mecánica mientras que la medición de difusión pulmonar de monóxido de carbono (DLCO) es la prueba más recomendada en la actualidad para evaluar el intercambio de gases a nivel pulmonar; cuando estas pruebas no son concluyentes para determinar la deficiencia respiratoria, habitualmente en la minoría de los casos, se debe realizar una prueba cardiopulmonar de ejercicio (PCPE).

Es importante mencionar que las PFR no son completamente específicas ni diagnósticas de una enfermedad, así como un resultado normal tampoco descarta una enfermedad. Las pruebas en reposo (espirometría y DLCO) pueden tener una correlación variable con la capacidad funcional real del paciente que sólo puede ser medida de manera definitiva por PCPE. Asimismo, el daño funcional puede ser he-

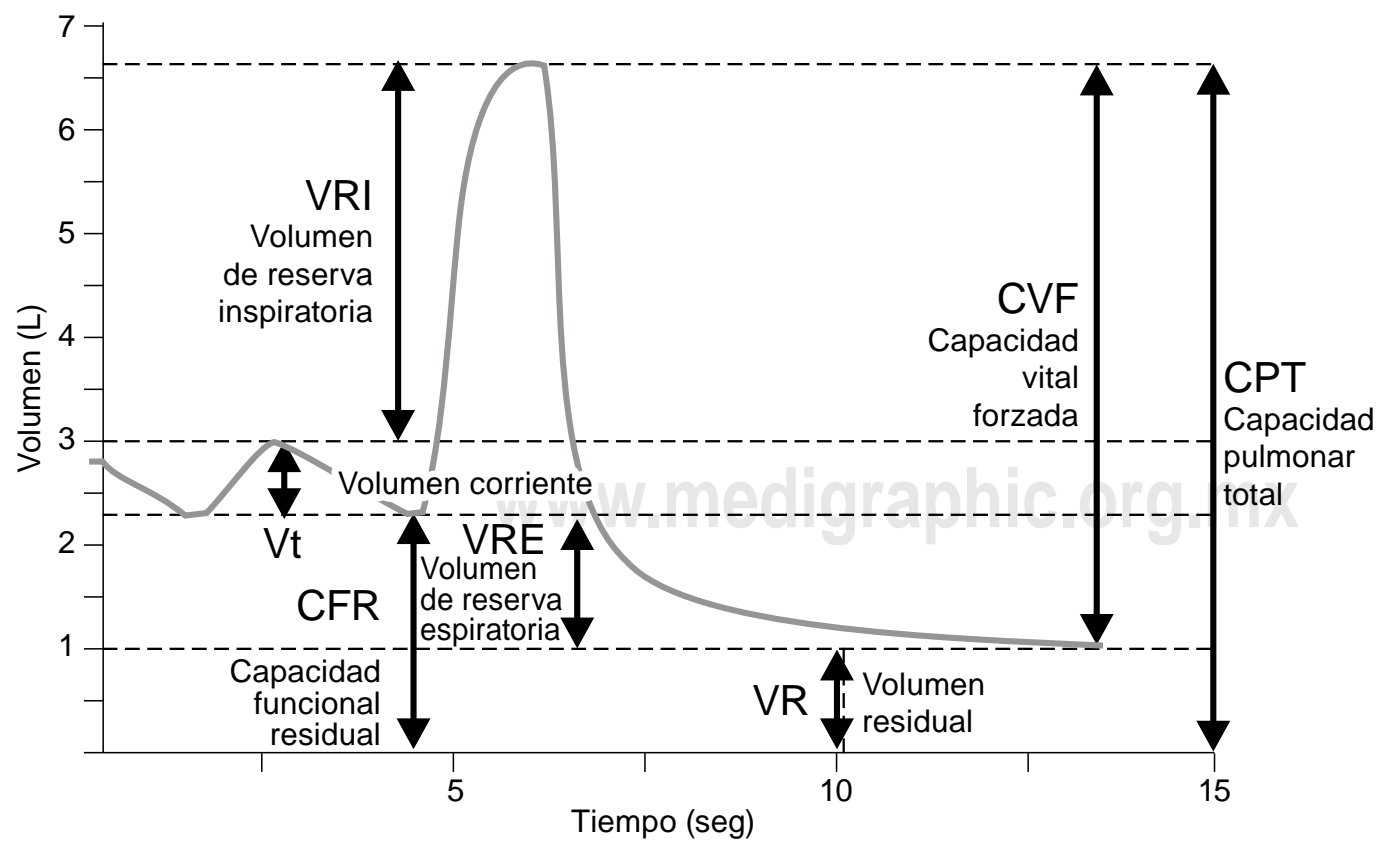

\section{Figura 1.}

Espirograma normal cronometrado. Se muestra cada uno de los volúmenes y capacidades que lo componen. La espirometría permite medir los más importantes, particularmente la capacidad vital forzada (CVF) que es una estimación del tamaño pulmonar. Por el contrario, no permite medir el volumen residual (VR) y en consecuencia la capacidad funcional residual (CFR) y la capacidad pulmonar total (CPT). 


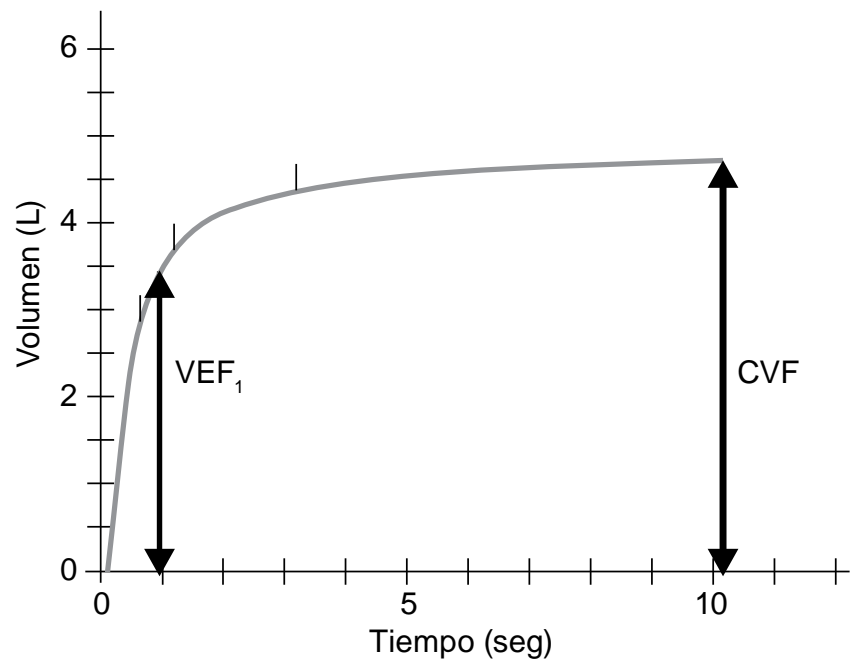

Figura 2. Curva volumen-tiempo de una espirometría normal. Se grafica el tiempo en segundo $(x)$ contra el volumen en el eje vertical $(y)$. La gráfica tiene un inicio muy vertical donde la mayor parte del volumen de aire se exhala en el primer segundo. Posteriormente, sigue una rodilla o transición donde el volumen tiende a caer y termina en una meseta cuando el individuo no exhala más aire, habitualmente después del segundo 6 . En la gráfica se identifican con facilidad la capacidad vital forzada (CVF) al final de la espiración y el volumen espiratorio forzado durante el primer segundo de la espiración $\left(\mathrm{VEF}_{1}\right)$.

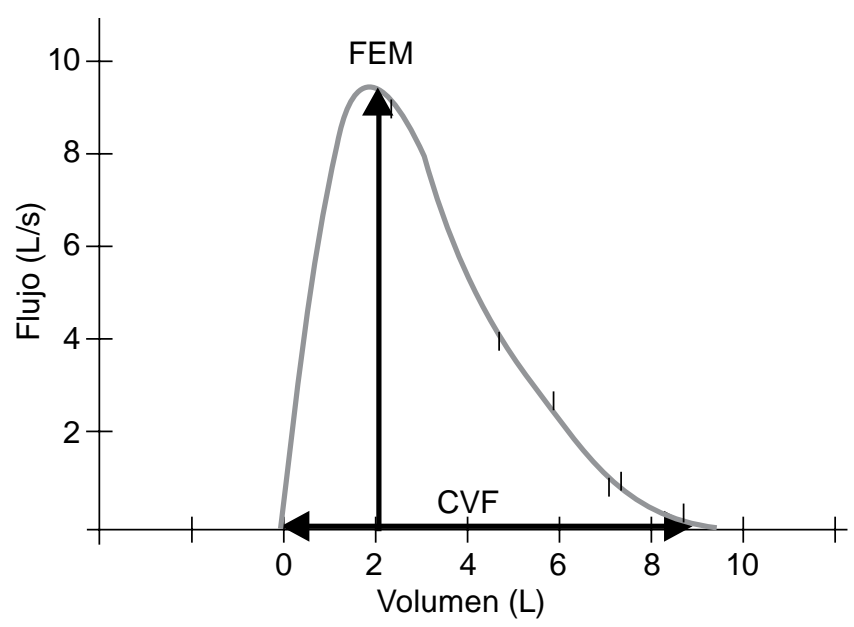

Figura 3. Curva flujo-volumen de una espirometría normal que corresponde a la misma prueba de la gráfica de la figura 2. Se grafica el volumen (eje horizontal) contra el flujo de aire que equivale a la aceleración del volumen. La curva tiene una fase espiratoria con un inicio ascendente pronunciado que termina en un pico que determina el flujo espiratorio pico o máximo (FEP o PEF, por sus siglas en inglés), continúa con una caída lineal del flujo hasta alcanzar la capacidad vital forzada (CVF). terogéneo entre enfermedades respiratorias y entre pacientes con la misma enfermedad. La valoración de deficiencia debería ser idealmente individualizada por enfermedad, pero esto sólo se ha hecho en el caso del asma.

\section{Espirometría}

En la figura 1 se muestra un espirograma normal cronometrado que ilustra los volúmenes y capacidades que pueden determinarse con las PFR. El volumen corriente $(\mathrm{Vt})$ es el volumen de aire que se moviliza durante un ciclo respiratorio en reposo. Si el individuo adicionalmente inhala el máximo volumen de aire posible o volumen de reserva inspiratoria (VRI), alcanza su capacidad pulmonar total (CPT). Posterior a ello, puede realizar una espiración forzada hasta alcanzar el máximo volumen de aire espirado; a este volumen de aire se le denomina capacidad vital forzada (CVF). El volumen de aire que permanece en los pulmones después de exhalar la CVF se llama volumen residual (VR). La suma del VR y el volumen de reserva espiratoria (VRE) representan la capacidad funcional residual (CFR) que es el volumen de aire que normalmente existe dentro del tórax en estado de reposo y que es una reserva para el intercambio gaseoso.

La espirometría forzada es una prueba cronometrada y mide el volumen o el flujo de aire que un individuo inhala o exhala en función del tiempo (figuras 1 a 3). El esfuerzo espiratorio es forzado o máximo y con frecuencia se le denomina maniobra de CVF. Además, la espirometría permite medir el volumen espiratorio forzado en el primer segundo $\left(\mathrm{VEF}_{1}\right)$. EI VEF $\mathrm{VE}_{1}$ es el volumen de aire exhalado durante el primer segundo de la maniobra de CVF y es el principal flujo espiratorio. Un adulto sano menor de 50 años puede exhalar $70 \%$ o más de su capacidad vital durante el primer segundo $\left(\mathrm{VEF}_{1} / \mathrm{CVF}>70 \%\right)$. La espirometría forzada es la prueba considerada estándar de oro para definir obstrucción al flujo aéreo. Cuando existen procesos obstructivos de la vía aérea tiende a disminuir $\mathrm{VEF}_{1}$ y la relación $\mathrm{VEF}_{1} / \mathrm{CVF}$. Los volúmenes medidos se expresan en litros $(L)$ y los flujos en litros por segundo (L/s), ambos en condiciones estándar de presión barométrica y temperatura corporal (BTPS, por sus siglas en inglés).

La espirometría debe incluir siempre las gráficas de volumen tiempo (figura 2) y de flujo volumen (figura 3); son de mucha utilidad para valorar la calidad de las maniobras y para fines de interpretación. La gráfica volumen tiempo presenta el tiempo en segundos en el eje horizontal (eje $\mathrm{x}$ ) contra el volumen en litros en el eje vertical (eje y). Un esfuerzo aceptable muestra un incremento brusco en el volumen durante el primer 
segundo de la espiración. Después, la curva hace una transición en forma de rodilla entre los segundos 1 y 2 $y$ al final una meseta donde no hay incremento significativo de volumen. La mayoría de los adultos alcanzan la CVF antes del segundo 6; sin embargo, algunos adultos mayores o personas con obstrucción al flujo aéreo requieren más de 10 segundos de espiración. En esta gráfica es fácil identificar la CVF, el VEF ${ }_{1}$ y el tiempo de exhalación forzada.

La gráfica flujo volumen (figura 3) presenta el volumen en el eje horizontal y el flujo aéreo en el eje vertical.

Tabla 5. Recomendaciones mínimas para estandarización de la espirometría.

Equipo:

1. Equipo seleccionado que cumpla las recomendaciones de desempeño técnico de la Sociedad Americana de Tórax (ATS) y la Asociación Europea Respiratoria (ERS)

2. Calibración o verificación de calibración del equipo el día en que se realiza la prueba

3. Debe permitir presentar e incluir en el informe de resultados las curvas de volumen-tiempo y flujo-volumen

Técnico:

1. Se recomienda técnico entrenado y certificado en espirometría en cursos especiales como en el del Instituto de Salud y Seguridad Ocupacional de los Estados Unidos (NIOSH) o de la Asociación Latinoamericana del Tórax (ALAT)

Prueba (maniobra):

1. Seguir las indicaciones y contraindicaciones de la prueba

2. Previo a la prueba el técnico debe explicar y demostrar la maniobra

3. Deben completarse tres buenas maniobras con esfuerzo máximo, con meseta espiratoria de al menos un segundo y tiempo espiratorio mínimo de 6 segundos desde los 10 años o más de edad

4. Esfuerzos libres de artefactos como terminación temprana, esfuerzo submáximo, tos, fugas del sistema o cierre glótico

5. La prueba debe ser repetible con una variación no mayor a $150 \mathrm{~mL}$ entre los dos mejores valores de CVF o VEF $_{1}$

Valores de referencia e interpretación:

1. Se recomienda usar valores de referencia recientes para población local o externos probados en la población

2. Interpretación de acuerdo con los estándares de la ATS/ ERS (2005)

Respuesta al broncodilatador

1. Administración de 200 a $400 \mu \mathrm{g}$ de salbutamol inhalado (cuatro dosis por separado con intervalos de 30 segundos), con cámara espaciadora, inhalación máxima y apnea de 5 a 10 segundos antes de volver a exhalar

2. Prueba positiva con cambio en $\operatorname{VEF}_{1}$ y/o CVF $>12 \%$ y un incremento de $>200 \mathrm{~mL}$ del valor basal
Esta curva es de gran utilidad para evaluar el esfuerzo inicial del paciente. Cuando la exhalación es con máximo esfuerzo, la fase espiratoria es de forma triangular, tiene un ascenso muy vertical y termina en pico llamado flujo espiratorio máximo o pico (FEM o FEP). La fase inspiratoria es semicircular y no siempre se presenta, ya que con frecuencia sólo se realiza la espiración. En esta curva se identifican la CVF en el eje horizontal y todos los flujos espiratorios, excepto el $\mathrm{VEF}_{1}$.

La espirometría es de gran utilidad para evaluar la salud y las enfermedades respiratorias, entre ellas las ERO, así como para monitorear evolución de una enfermedad y la respuesta a intervenciones, como los tratamientos. Además, sirve para valorar riesgos y exposiciones ambientales y correlaciona bien con la morbilidad y la esperanza de vida..$^{10-12}$ Es la prueba de función pulmonar mejor estandarizada y la más reproducible, por lo que es la más confiable; no obstante, debe cumplir con las recomendaciones mínimas de estandarización (tabla 5). Al presente, existen los procedimientos detallados tanto para su realización bajo estricto control de calidad como para su interpretación. ${ }^{12-18}$

Un panel de expertos internacionales auspiciado por la Asociación Americana del Tórax (ATS) y la Sociedad Europea Respiratoria (ERS) ha publicado las recomendaciones vigentes de estandarización de espirometría (estándares ATS/ERS 2005), las cuales se resumen en la tabla $5 .{ }^{12}$ Es fundamental que los laboratorios de función pulmonar y las personas que realizan estas pruebas cumplan con estas recomendaciones. Sólo los espirómetros fabricados en los últimos años reúnen o superan las recomendaciones técnicas. El equipo debe tener un control de calidad periódico y es indispensable que cumpla una calibración o una verificación de calibración el día en que se realiza la prueba. Los técnicos deben ser entrenados y certificados para la prueba y para ello existen cursos especializados con reconocimiento internacional, como el certificado por el Instituto de Salud y Seguridad Ocupacional de los Estados Unidos (NIOSH) y por la Asociación Latinoamericana del Tórax (ALAT). En México, estos cursos son programados con frecuencia en el Instituto Nacional de Enfermedades Respiratorias Ismael Cosío Villegas y en la sede de la Sociedad Mexicana de Neumología y Cirugía de Tórax (SMNyCT). En cuanto a la prueba se deben cumplir todos los pasos de instrucción y demostración del esfuerzo al paciente y se deben completar tres maniobras aceptables y repetibles. Para la interpretación se deben usar valores de referencia generados bajo estándares actuales; en general, se prefieren valores de población local o externos validados en la población. Para población mexicana adulta existen tres valores predichos o de referencia que han sido propiamente publicados y que 


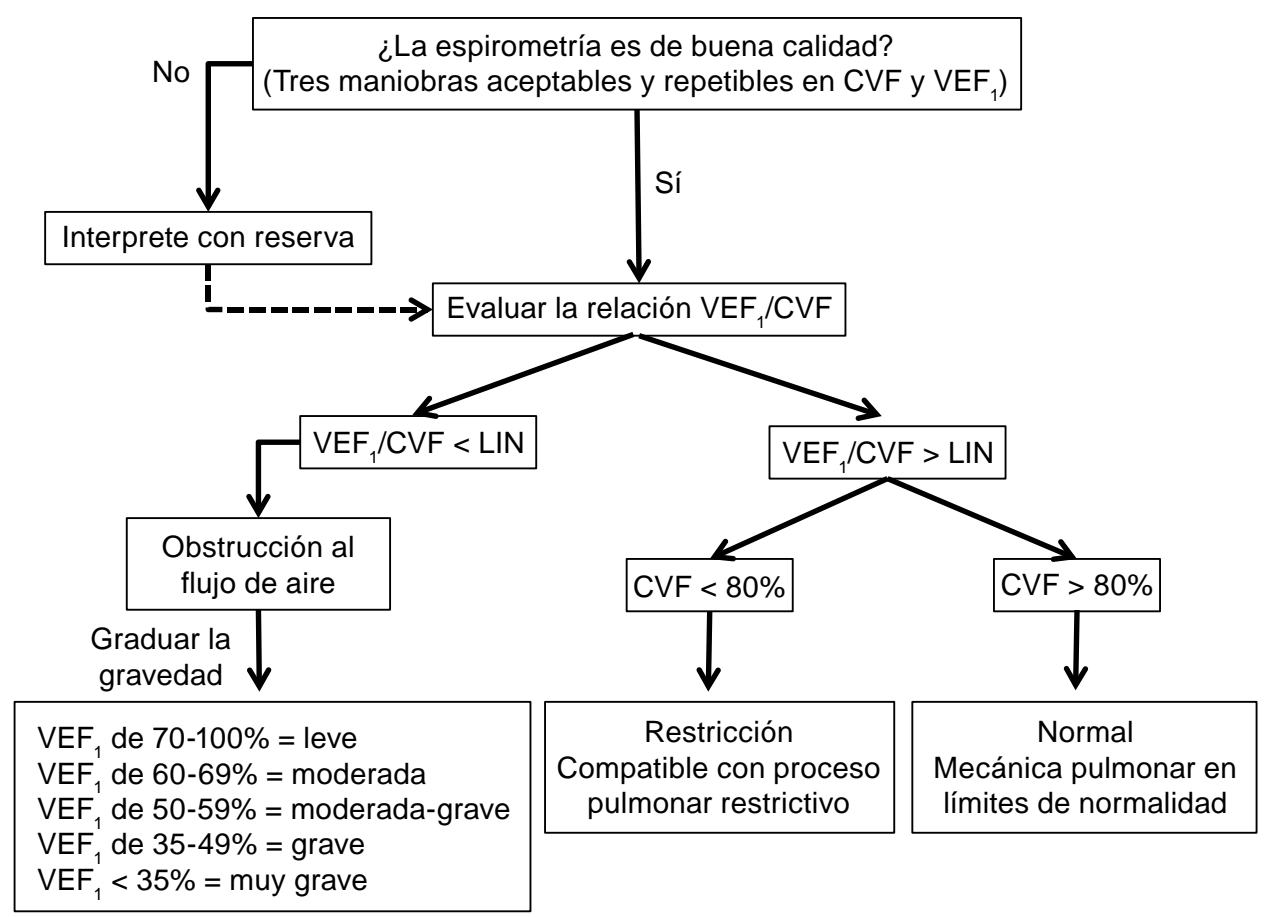

Figura 4. Diagrama de flujo recomendado para el proceso de interpretación de la espirometría. El primer paso recomendado es evaluar la calidad de la prueba de acuerdo a los criterios de aceptabilidad y repetibilidad. Para determinar el patrón ventilatorio se debe iniciar la interpretación con la evaluación de la relación $\mathrm{VEF}_{1} / \mathrm{CVF}$. En términos generales debe ser mayor al $70 \%$ para personas menores de 50 años. Si la relación es menor al límite inferior normal (LIN) el cual depende de la edad (Tabla 6), la prueba se interpreta como diagnóstica de obstrucción al flujo de aire; posteriormente, debe graduarse la gravedad o el grado de obstrucción. Una relación $V E F / / C V F$ normal (>LIN) con CVF mayor o igual al $80 \%$ define una espirometría normal (mecánica pulmonar normal) mientras que una relación VEF1/CVF normal con una CVF menor del $80 \%$ es compatible con un proceso restrictivo pulmonar (pulmón pequeño).

Tabla 6. Límites inferiores de normalidad (percentil 5) para la relación $\mathrm{VEF}_{1} / \mathrm{CVF}$ de acuerdo con la edad.

\begin{tabular}{ccc}
\hline & \multicolumn{2}{c}{ VEF $_{1} /$ CVF $(\%)$} \\
\cline { 2 - 3 } Edad & Mujeres & Hombres \\
\hline 40 & 72 & 70 \\
50 & 70 & 68 \\
60 & 67 & 66 \\
70 & 65 & 64 \\
80 & 63 & 62 \\
\hline
\end{tabular}

corresponden a las ecuaciones referidas para población México-americana y mexicana. ${ }^{19-21}$

La interpretación de la prueba debe realizarse por un profesional entrenado y siguiendo los lineamientos internacionales para la determinación del patrón funcional y la graduación de gravedad en caso de anormalidad. 13,16-18 La figura 4 ilustra el diagrama de flujo recomendado para la interpretación estandarizada de acuerdo con las recomendaciones de ATS/ERS 2005. ${ }^{13}$ La interpretación inicia con el análisis de la calidad técnica de la prueba. El patrón ventilatorio se determina con la evaluación inicial de la relación $\mathrm{VEF}_{1} / \mathrm{CVF}$; si esta relación es menor al límite inferior normal (LIN), la prueba se interpreta como diagnóstica de obstrucción al flujo de aire. EI LIN depende de la edad (tabla 6), pero en términos generales debe ser mayor al $70 \%$ para personas menores de 50 años. Si la prueba confirma obstrucción, posteriormente se debe graduar la gravedad de acuerdo al porcentaje del predicho del $\mathrm{VEF}_{1}$ (figura 4). Una relación $\mathrm{VEF}_{1} / \mathrm{CVF}$ normal (> LIN) con CVF mayor o igual al $80 \%$ define una espirometría normal (mecánica pulmonar normal) mientras que una relación $\mathrm{VEF}_{1} / \mathrm{CVF}$ normal con una CVF menor del $80 \%$ es compatible con un proceso pulmonar restrictivo (volumen pulmonar disminuido).

\section{Espirometría con broncodilatador}

Esta prueba es de utilidad para evaluar cambios en el volumen pulmonar (CVF) y en los flujos espiratorios 
$\left(\mathrm{VEF}_{1}\right)$ posterior a la administración de un medicamento broncodilatador por vía inhalada ${ }^{13,16,18}$ En términos generales, no se debe haber inhalado broncodilatadores de 4 a 24 horas antes de la prueba (de acuerdo a la vida media de cada medicamento). La respuesta fisiológica al broncodilatador integra el epitelio bronquial, nervios, mediadores y músculo liso de la vía aérea. Para realizar la prueba se recomienda completar una espirometría basal que cumpla las recomendaciones mínimas (tabla 5). A continuación se administran 200 a $400 \mu \mathrm{g}$ (2 a 4 inhalaciones) de salbutamol, usando cámara espaciadora. La espirometría se repite 15 minutos después, una respuesta al broncodilatador se considera positiva cuando la CVF y/o el VEF ${ }_{1}$ mejoran más del $12 \%$ y más de $200 \mathrm{~mL}$ con respecto al valor basal.

La prueba de broncodilatador siempre es de mucha utilidad en una espirometría diagnóstica. Un proceso obstructivo reversible inmediatamente con el broncodilatador o tardíamente (días o semanas) de manera espontánea o con tratamiento, habitualmente es compatible con asma, mientras que un proceso obstructivo no completamente reversible es típico de enfermedades con obstrucción al flujo aéreo fija y crónica, como es el caso de la EPOC.

\section{Difusión pulmonar de monóxido de carbono}

La medición de la difusión o transferencia de monóxido de carbono (DLCO o TLCO) es una prueba de intercambio gaseoso que mide con relativa facilidad el proceso de oxigenación a nivel alvéolo-capilar. ${ }^{22-24}$ La prueba es muy sencilla y toma unos 15 a 20 minutos realizarla. A diferencia de la espirometría, no requiere de maniobras forzadas por lo que puede resultar más cómoda. Para esta prueba se usa como marcador de oxigenación el monóxido de carbono (CO) que pasa libremente la membrana y que es altamente afín a la hemoglobina. La cantidad de $\mathrm{CO}$ administrada es muy pequeña y su transferencia se calcula a partir de la relación entre el volumen de $\mathrm{CO}$ inspirado y espirado.

El estudio de la difusión se basa en la Ley de Fick que describe que el paso de un gas a través de una membrana es directamente proporcional a la superficie de la membrana, la diferencia de concentración del gas entre los dos lados de la membrana y es inversamente proporcional a su grosor. La superficie de la membrana alvéolo-capilar es en promedio de $70 \mathrm{~m}^{2}$ y su grosor promedio es de $5 \mu \mathrm{m}$. Asimismo, el coeficiente de difusión del gas es directamente proporcional a su solubilidad e inversamente proporcional a su peso molecular; por ejemplo, el $\mathrm{CO}_{2}$ difunde unas 20 veces más rápido que el $\mathrm{O}_{2}$ ya que es mucho más soluble y su peso molecular no es mucho mayor. Además, la

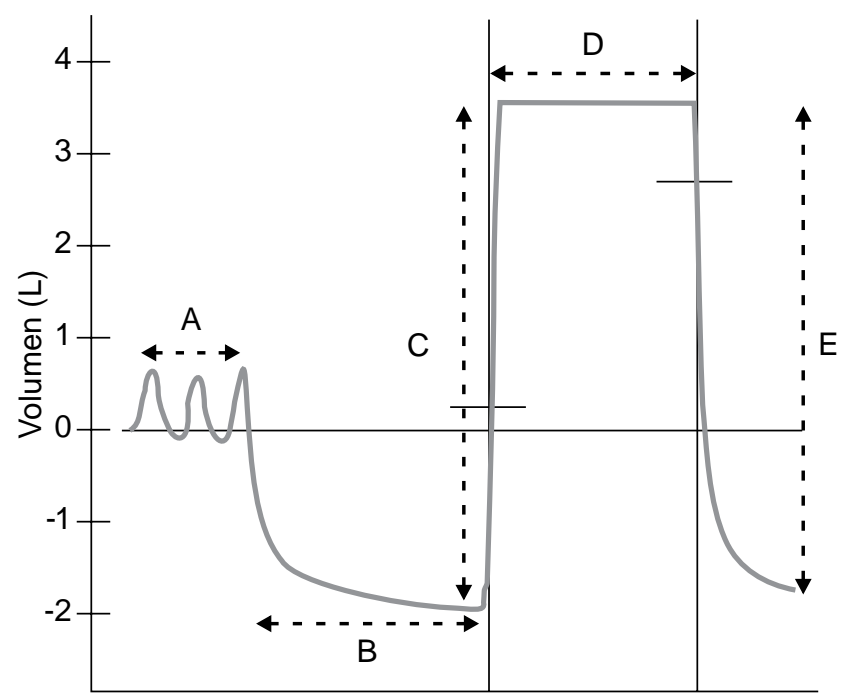

Tiempo (s)

Figura 5. Maniobra respiratoria correspondiente a la prueba de difusión pulmonar de monóxido de carbono de respiración única (DLCO). La maniobra inicia con respiraciones en volumen corriente $(A)$, seguidas de una maniobra de volumen de reserva espiratoria hasta llegar a volumen residual (B). Después, se realiza con inspiración máxima $(\mathrm{C})$ o volumen inspiratorio $\left(\mathrm{V}_{\text {in }}\right)$ y que debe de ser $>85 \%$ de la mayor CVF, durante esta inhalación se libera el gas de prueba que contiene $0.3 \%$ de monóxido de carbono (CO) y un gas inerte que suele ser helio, neón o metano, el cual permite medir el volumen de aire ventilable en el pulmón. Posteriormente, se realiza un período de apnea de 10 segundos de duración (D) que es donde se transfiere el CO hasta la hemoglobina; al final se realiza una espiración mayor a 4 segundos (E) que es donde se capta el aire espirado proveniente de los alvéolos para calcular la proporción de CO transferido a la hemoglobina.

capacidad de difusión del $\mathrm{O}_{2}$ depende de la cantidad de hemoglobina disponible y el tiempo de exposición del eritrocito a los alvéolos que en reposo es de 0.7 a 1.2 segundos. En términos generales, la prueba de DLCO mide la integridad de la membrana alvéolo-capilar; las enfermedades que disminuyen la superficie de la membrana, como el enfisema pulmonar o que la alteran por inflamación o fibrosis, como las neumoconiosis, cursan con una DLCO proporcionalmente más baja de acuerdo al grado de daño alveolar.

La prueba de DLCO ha sido estandarizada internacionalmente (ATS/ERS 2005). ${ }^{24}$ Recomendaciones recientemente actualizadas, ${ }^{25}$ por lo que se sugiere seguir estos procedimientos para la técnica de una sola respiración. ${ }^{24,26}$ La maniobra (figura 5) consiste en una exhalación máxima hasta llegar a volumen residual; posteriormente, se realiza con inspiración máxima equivalente a una capacidad vital inspiratoria 
o volumen inspiratorio (Vin), durante esta inhalación se libera el gas de prueba que contiene $0.3 \%$ de $\mathrm{CO}$ y un gas inerte que suele ser helio, neón o metano, el cual permite medir el volumen de aire ventilado en el pulmón. Después se realiza un período de apnea de 10 segundos de duración que es donde se transfiere el CO hasta la hemoglobina; finalmente, se realiza una espiración mayor a 4 segundos para captar el aire espirado proveniente de los alvéolos para calcular la proporción de $\mathrm{CO}$ transferido a la hemoglobina. La prueba completa consiste en dos mediciones consecutivas y que muestren una diferencia no mayor a tres unidades $(\mathrm{mmol} / \mathrm{min} / \mathrm{kPa}$ ) o $10 \%$.

La interpretación de la prueba también se realiza a partir de una comparación directa con los valores normales o predichos. Los estándares internacionales vigentes ${ }^{25}$ sólo recomiendan las ecuaciones de referencia generadas bajo los estándares previos (ATS/ERS 2005). Recientemente, se han publicado ecuaciones de referencia para población latinoamericana que incluye mexicanos y que están ajustadas por altitud y hemoglobina. ${ }^{27} \mathrm{Al}$ igual que flujos y volúmenes pulmonares, una disminución de la DLCO se define cuando está por debajo del LIN (aproximadamente $80 \%$ del predicho). Para graduar la gravedad se recomienda definir disminución leve con $D_{L} ; C O \geq 60 \%$ y $<$ LIN , disminución moderada de 40 a $59 \%$ y grave cuando es menor al $40 \%$ del predicho.

\section{Prueba cardiopulmonar de ejercicio}

Las pruebas de función respiratoria convencionales en reposo suelen diagnosticar y graduar la gravedad de la mayoría de las enfermedades respiratorias crónicas. Aunque no dan información directa de la capacidad funcional del individuo, por lo que las pruebas de ejercicio, particularmente la PCPE puede tener un impacto en el proceso de decisión clínica para identificar origen de síntomas (disnea) y la contribución de diferentes órganos (pulmones, corazón o musculoesquelético) en la limitación al ejercicio. ${ }^{28,29}$ La PCPE puede ser requerida en una minoría de pacientes con ERO y permite definir de manera definitiva el grado de funcionalidad del individuo, ya que mide la capacidad de realizar trabajo físico; en consecuencia, mejora la precisión con que se evalúa el grado de deficiencia funcional.

La PCPE se realiza en un cicloergómetro o en una banda sinfín con protocolos de incremento progresivos de trabajo. Durante la prueba se mide el consumo de oxígeno $\left(\mathrm{VO}_{2}\right)$, la producción de $\mathrm{CO}_{2}\left(\mathrm{VCO}_{2}\right)$ y la ventilación minuto; también se pueden monitorear otras variables de interés durante el ejercicio, como el electrocardiograma, la presión arterial y la saturación de oxígeno por pulsooximetría $\left(\mathrm{SpO}_{2}\right)$. Asimismo, si se obtienen gasometrías arteriales se agrega información sobre el intercambio gaseoso durante el ejercicio.

La PCPE realizada en cicloergómetro electrónicamente controlado es preferible a la realizada en banda sinfín; es menos costoso y más seguro, permite medir directamente el trabajo realizado con menos artefactos en el ECG y facilita la toma de muestras de sangre arterial. Los protocolos de ejercicio son variados; se recomiendan incrementos de trabajo de 5 a 25 watts por minuto y la capacidad aeróbica se debe alcanzar en menos de 12 minutos, ya que la prueba resulta incómoda debido a la instrumentación.

El consumo máximo de oxígeno ( $\mathrm{VO}_{2}$ máx) se alcanza cuando existe meseta en el $\mathrm{VO}_{2}$, es decir, no aumenta a pesar de incrementos en el trabajo. Cuando no se alcanza meseta en el $\mathrm{VO}_{2}$ se denomina como consumo pico de oxígeno ( $\mathrm{VO}_{2}$ pico); para fines prácticos ambos parámetros son intercambiables y representan el mejor indicador de la capacidad de ejercicio. Estos parámetros deben informarse como valores absolutos (L/min) y como porciento del predicho. Un $\mathrm{VO}_{2}$ máx normal refleja capacidad aeróbica y capacidad de ejercicio normales y descarta anormalidad funcional. En contraste, un $\mathrm{VO}_{2}$ máx disminuido traduce pobre esfuerzo o problemas en la entrega de $\mathrm{O}_{2}$ que pueden tener origen en los pulmones, circulación sistémica y pulmonar, musculoesquelético o en el trasporte sanguíneo.

\section{Evaluación de la deficiencia respiratoria}

Varias organizaciones profesionales internacionalmente reconocidas han publicado sus recomendaciones para la evaluación de la deficiencia respiratoria. La ATS publicó en 1986 en conjunto con la Asociación Americana del Pulmón (ALA) las recomendaciones para evaluación de deficiencia y discapacidad secundaria a enfermedades respiratorias..$^{30}$ Estas recomendaciones no han sido actualizadas de manera reciente, excepto para el caso de asma, revisada y publicada en 1993.31 La Sociedad Europea de Fisiología Respiratoria Clínica (SEPCR) publicó sus recomendaciones para graduar discapacidad respiratoria en $1990 .{ }^{32}$ Finalmente, la Asociación Médica Americana (AMA) en sus guías para evaluación de deficiencia que fueron revisadas en el año 2000, incluyen recomendaciones específicas para valoración del sistema respiratorio. ${ }^{33}$ Las recomendaciones de las tres organizaciones son muy similares, sin embargo, las de la AMA son las que con mayor frecuencia se utilizan y refieren en la literatura. Estas valoraciones son secuenciales como se describió. Si con la prueba más sencilla, la espirometría, se 
puede documentar una alteración funcional grave, ya no es necesario hacer pruebas adicionales. Se han promovido esquemas de evaluación «en paquete», que someten a los solicitantes a espirometría, DLCO, PCPE y quizá otras pruebas, pero el procedimiento en muchas ocasiones es redundante y además incrementa los costos. Es más eficiente en forma secuencial y las pruebas más sofisticadas pueden realizarse sólo en algunos centros especializados, ya que se requerirán en menos individuos.

La valoración médica de deficiencia respiratoria debe incluir una valoración clínica previa con historia médica y examen físico cuidadoso, radiografía de tórax y PFR estandarizadas que siempre deben incluir de inicio a la espirometría. Todas las recomendaciones publicadas se enfocan casi siempre en evaluar el grado de deficiencia respiratoria relacionada a una reducción de la función pulmonar, lo cual se asocia a una alta probabilidad de limitación para la capacidad de realizar ejercicio físico. Antes de la evaluación de deficiencia es recomendable que ya exista un diagnóstico médico establecido, ya que la función pulmonar puede estar disminuida directamente por enfermedades no respiratorias, como deformidades musculoesqueléticas del tórax, enfermedades neuromusculares o incluso por insuficiencia cardíaca. Las pruebas de función respiratoria deben realizarse con el paciente estable y siguiendo todas las recomendaciones de estandarización previamente referidas.
Las guías de la ATS, SEPCR y AMA recomiendan realizar la espirometría y prueba de DLCO en todos los casos y sólo una minoría de pacientes requiere de una PCPE. En el proceso de valuación se incluyen sólo los valores predichos de CVF, VEF y de DLCO. La SEPC y la AMA insisten en utilizar límites inferiores de normalidad de acuerdo con la distribución del valor en la población de referencia. La AMA especifica grados de deficiencia respiratoria de acuerdo a valores $\mathrm{VO}_{2}$ máx $(\mathrm{mL} / \mathrm{kg} / \mathrm{min})$ y trabajo realizado (Mets). La ATS y la SEPCR gradúan la deficiencia pulmonar como: no deficiencia y deficiencia leve, moderada o grave. Éstos son, respectivamente, equivalentes a las clases 1,2 , 3 y 4 de la AMA.

\section{Propuesta de evaluación de deficiencia respiratoria en México}

La propuesta del grupo de trabajo de la STPS para la graduación de la deficiencia respiratoria en presencia de ERO se resume en la tabla 7 y en la figura 6 . En general, estos procedimientos son congruentes con las recomendaciones internacionales previamente descritas. ${ }^{30-33} \mathrm{La}$ figura 6 muestra el diagrama de flujo recomendado para la evaluación funcional respiratoria en el proceso de graduación de la deficiencia respiratoria. Éste es un esquema de uso progresivo de las pruebas de acuerdo con su complejidad y por lo tanto su disponibilidad, lo que debe facilitar y optimizar su

Tabla 7. Graduación de deficiencia respiratoria.

\begin{tabular}{|c|c|c|c|c|c|}
\hline Grado & $\begin{array}{c}\text { Espirometría } \\
\text { VEF }_{1} \text { o CVF (\%) }\end{array}$ & $\begin{array}{l}\text { Transferencia (difusión) } \\
\text { pulmonar de monóxido } \\
\text { de carbono }\end{array}$ & $\begin{array}{c}\text { Consumo máximo de } \\
\text { oxígeno en la prueba } \\
\text { de ejercicio cardiopul- } \\
\text { monar } \\
\mathrm{VO}_{2} \text { máx (mL/kg min) }\end{array}$ & Pronóstico & $\begin{array}{c}\text { Valuación (\% de } \\
\text { incapacidad } \\
\text { permanente) }\end{array}$ \\
\hline 1 & $\geq 80$ & $\geq 80$ & $\geq 25$ & $\begin{array}{l}\text { Bueno para la vida y } \\
\text { para la función }\end{array}$ & $5-10$ \\
\hline 2 & $65-79$ & $65-79$ & $20-25$ & $\begin{array}{l}\text { Bueno para la vida } \\
\text { y reservado para la } \\
\text { función }\end{array}$ & $11-25$ \\
\hline 3 & $51-64$ & $51-64$ & $15-20$ & Reservado para la & $26-60$ \\
\hline 4 & $\leq 50$ & $\leq 50$ & $<15$ & $\begin{array}{c}\text { Malo para la vida y } \\
\text { para la función }\end{array}$ & 61 a 100 \\
\hline
\end{tabular}

1. La evaluación funcional debe iniciar con espirometría como prueba de mecánica respiratoria, seguida de DLCO como prueba de intercambio gaseoso para valorar transferencia de oxígeno a nivel pulmonar. En caso de existir duda se recomienda prueba de ejercicio cardiopulmonar. 2. La incapacidad funcional es muy grave si cualquier valor de $\mathrm{VEF}_{1}, \mathrm{CVF}_{\mathrm{o}} \mathrm{DLCO}$ está por abajo del $30 \%$ del esperado o cuando la VO máx es menor a $10 \mathrm{~mL} / \mathrm{kg} / \mathrm{min}$

3. El porcentaje de valuación lo determina la prueba de función respiratoria con el valor más bajo encontrado.

4. La valuación en grado 1 representa deficiencia orgánica, por ejemplo, afectación radiológica en presencia de pruebas de función respiratoria en límites normales. 
Paso 1: Espirometría (todos los pacientes)

Paso 2: DLCO

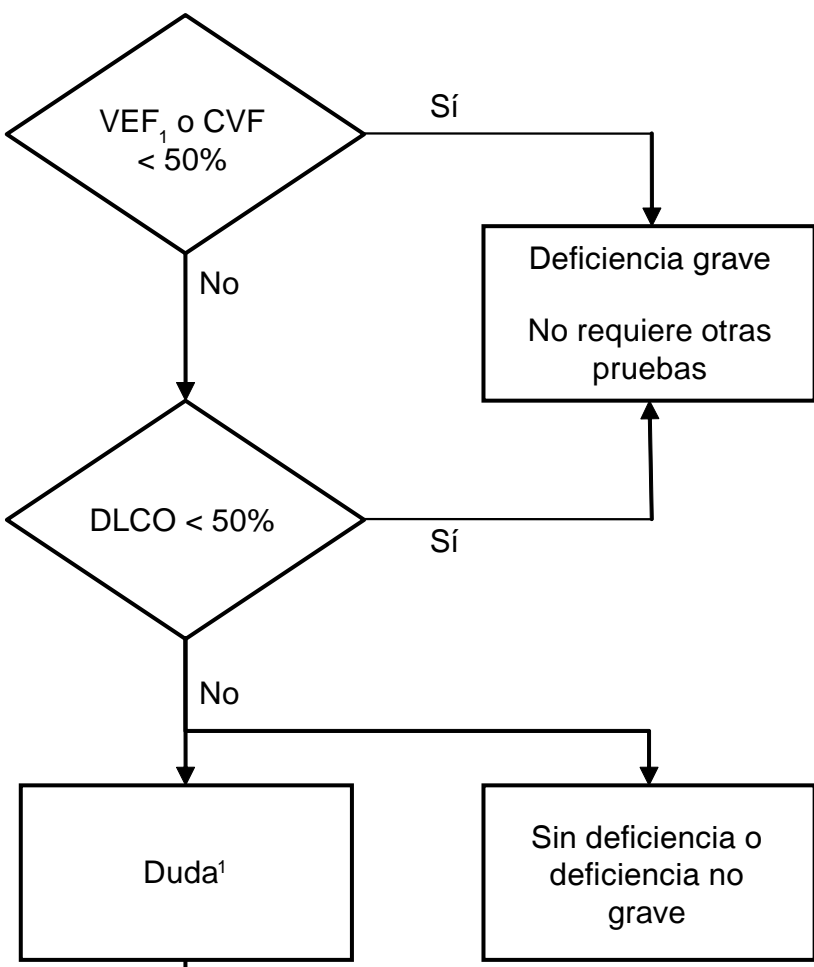

${ }^{1}$ Disnea o limitación referida excesiva para la alteración en espirometría o DLCO.

Figura 6.

Diagrama de flujo recomendado para la evaluación funcional respiratoria en el proceso de graduación de la deficiencia respiratoria con el uso progresivo de prueba de función respiratoria. Este esquema sugerido busca disminuir el número de pruebas que se realizan en los centros de referencia. El procedimiento inicia en todo enfermo con la realización de una espirometría. Si la prueba confirma un valor de $\mathrm{VEF}_{1}$ 。 CVF igual o menor al $50 \%$ se determina deficiencia grave sin requerir de mayor evaluación. El segundo paso requiere valorar el intercambio de gases a nivel pulmonar con la prueba de DLCO. En la mayoría de los enfermos sería posible graduar el estado de deficiencia respiratoria sólo con espirometría y DLCO. En caso de duda, principalmente con la presencia de disnea o limitación referida excesiva para las alteraciones encontrada en la espirometría o DLCO se recomienda una prueba de ejercicio pulmonar para determinar el consumo de oxígeno $\left(\mathrm{VO}_{2}\right.$ máx para determinación definitiva del

No requiere otras pruebas grado de deficiencia.

utilización. La valoración funcional y la graduación de la deficiencia respiratoria siempre deben iniciar con la espirometría. Si la prueba confirma un valor de $\mathrm{VEF}_{1}$ o CVF igual o menor al $50 \%$ se determina deficiencia grave sin requerir de otras pruebas necesariamente. El segundo paso requiere valorar el intercambio de gases a nivel pulmonar con la prueba de DLCO. En la mayoría de los enfermos debe ser posible graduar el estado de deficiencia respiratoria sólo con la espirometría y la prueba de DLCO. En caso de existir duda, por resultados limítrofes y principalmente con la presencia de disnea o limitación referida excesiva para las alteraciones encontradas en la espirometría o la DLCO, es recomendable que se complete una PECP para medir $\mathrm{VO}_{2}$ máx y determinar de manera definitiva el grado de deficiencia.

En la tabla 7 se especifican los grados de deficiencia respiratoria de acuerdo con los resultados de las PFR, el pronóstico correspondiente y el porcentaje de valuación de la deficiencia respiratoria, como lo establece la LFT. El grado de deficiencia está determinado por el valor más bajo encontrado en cualquiera de la PFR que 
Tabla 8. Graduación de deficiencia respiratoria en asma y disfunción reactiva de la vía aérea.

\begin{tabular}{|c|c|c|c|}
\hline $\begin{array}{l}\text { Función pulmonar (patrón de } \\
\text { obstrucción pulmonar) }\end{array}$ & Tratamiento establecido & Pronóstico & $\begin{array}{l}\text { Valuación (\% de } \\
\text { incapacidad } \\
\text { permanente) }\end{array}$ \\
\hline $\begin{array}{c}\mathrm{VEF}_{1}>80 \% \text { o variabilidad de } \\
\text { FEP }<20 \% \\
\text { Deficiencia mínima }\end{array}$ & $\begin{array}{l}\text { Broncodilatadores de corta duración } \\
\text { de uso ocasional }\end{array}$ & $\begin{array}{l}\text { Bueno para la vida y la } \\
\text { función }\end{array}$ & 5 a 25 \\
\hline $\begin{array}{c}\text { VEF }_{1} \text { de } 65-79 \% \text { o variabilidad } \\
\text { de FEP de } 20-29 \% \\
\text { Deficiencia leve }\end{array}$ & $\begin{array}{c}\text { Uso cotidiano de broncodilatadores } \\
\text { de larga duración y esteroides } \\
\text { inhalados a dosis bajas }\end{array}$ & $\begin{array}{l}\text { Bueno para la vida } \\
\text { y reservado para la función }\end{array}$ & 26 a 50 \\
\hline $\begin{array}{c}V E_{1} \text { de } 50-60 \% \text { o variabilidad } \\
\text { de FEP de } 20 \text { a } 29 \% \\
\text { Deficiencia moderada } \\
\end{array}$ & $\begin{array}{c}\text { Uso cotidiano de broncodilatadores } \\
\text { de larga duración y esteroides } \\
\text { inhalados a dosis altas }\end{array}$ & $\begin{array}{l}\text { Reservado para la vida y } \\
\text { reservado para la función }\end{array}$ & 51 a 75 \\
\hline $\begin{array}{c}\mathrm{VEF}_{1}<50 \% \text { o variabilidad de } \\
\text { FEP }>40 \% \\
\text { Deficiencia grave }\end{array}$ & $\begin{array}{l}\text { Uso cotidiano de broncodilatadores } \\
\text { de larga duración y esteroides } \\
\text { inhalados a dosis altas y esteroides } \\
\text { sistémicos orales }\end{array}$ & $\begin{array}{l}\text { Reservado para la vida y } \\
\text { malo para la función }\end{array}$ & 76 a 100 \\
\hline
\end{tabular}

integra el procedimiento. Los grados propuestos son del 1 al 4, que corresponde a un estado funcional en límites normales (Grado 1) o un estado funcional disminuido en grados del 2 al 4 que proporcionalmente corresponden a una condición funcional levemente disminuida (Grado 2), moderadamente disminuida (Grado 3) o gravemente disminuida (Grado 4).

Una condición especial de evaluación de deficiencia respiratoria corresponde al asma y al síndrome de disfunción reactiva de la vía aérea. Estas enfermedades son complicadas y se han descrito recomendaciones de evaluación y graduación de acuerdo con puntajes específicos que involucran resultados de espirometría, requerimientos de medicamentos y reversibilidad en la obstrucción e hiperreactividad bronquial ${ }^{23}$ Es decir, una persona con asma puede estar bien controlado y con función normal o cercana a la normal, debido al uso de varios medicamentos inhalados y de corticosteroides sistémicos a dosis altas; sin embargo, esto implica un mayor riesgo de eventos adversos y se considera con mayor incapacidad. En la tabla 8 se muestran los criterios de graduación de la deficiencia respiratoria y su valuación correspondiente en estas enfermedades.

\section{Consideraciones adicionales y perspectivas futuras}

Previamente se han revisado estándares internaciones de PFR y recomendaciones de graduación de deficiencia. Empero, existen diversas limitaciones que deben considerarse en nuestro país y algunos puntos de oportunidad para desarrollo de investigación en nuestro medio.
La evaluación funcional y la determinación de la deficiencia respiratoria por medio de las PFR se recomiendan hace muchos años. Aunque la disponibilidad de las pruebas, incluyendo la espirometría, es muy limitada, mientras que la prueba de DLCO y la PCPE son casi inexistentes, aun en las instituciones encargadas de evaluar y decretar discapacidad laboral. La infraestructura física relacionada con laboratorios de referencia que funcionen como centros de valuación será una necesidad de mediano y largo plazo.

En general existe poca difusión y enseñanza en el ambiente en las especialidades de Neumología, Medicina del Trabajo y Medicina Familiar en cuanto a la forma de evaluar la deficiencia y la discapacidad respiratoria.

Como es de esperarse, la actualización de la tabla de enfermedades de origen ocupacional y de los procedimientos de valuación conlleva el reto que requiere de la creación de la infraestructura física, así como la capacitación y la formación de nuevos recursos humanos que garanticen la adecuada implementación de los procedimientos propuestos.

\section{REFERENCIAS}

1. Secretaría del Trabajo y Previsión Social. Ley Federal del Trabajo. 14ª edición, México, D.F.; 2007.

2. Gulati M, Redlich CA. Occupational lung disorders. General principles and approaches. In: Fishman AP, editor. Pulmonary diseases and disorders. Fourth edition. New York, NY: McGraw Hill Medical; 2008. p. 933-942.

3. Blanc PD. Occupation and COPD: a brief review. J Asthma 2012;49(1):2-4. doi: 10.3109/02770903.2011.611957.

4. Organización Mundial de la Salud. Clasificación Internacional del Funcionamiento, de la Discapacidad y de la Salud: CIF. Ginebra: OMS; 2001. 
5. Taiwo OA, Cain HC. Pulmonary impairment and disability. Clin Chest Med 2002;23(4):841-851.

6. Gibson GJ. Spirometry: Then and now. Breath 2005;1(3):207-216.

7. West JB. Fisiología respiratoria. 6a edición. México: Editorial Médica Panamericana; 2002.

8. Vázquez-García JC. Fisiología respiratoria. En: CanoValle F, Ibarra-Pérez C, Morales-Gómez J, editores. Enfermedades respiratorias. Temas selectos. Madrid, España: Elsevier; 2006. p. 29-41.

9. Vázquez-García JC, Rojas-González A. Fisiología respiratoria. En: Cano-Valle F, editor. Enfermedades respiratorias. México, D.F.: Méndez Editores; 2008. p. 35-50.

10. Crapo RO. Pulmonary function testing. N Engl J Med 1994;331(1):25-30.

11. Chupp GL. Pulmonary function testing. Clin Chest Med 2001;22:759-767.

12. Miller MR, Hankinson J, Brusasco V, et al; ATS/ERS Task Force. Standardisation of spirometry. Eur Respir J 2005;26(2):319-338.

13. Pellegrino R, Viegi G, Brusasco V, et al. Interpretative strategies for lung function tests. Eur Respir J 2005;26(5):948-968.

14. Wanger J, Crapo RO, Irvin CG. Pulmonary function laboratory management and procedures manual. A project of the American Thoracic Society. NY: ATS.

15. Pérez-Padilla JR, Vázquez-García JC. Manual de entrenamiento en espirometría. Asociación Latinoamericana del Tórax (ALAT), 2004. Fecha de consulta: junio 26, 2012. Disponible en: http://www. alatorax.org.

16. Vázquez-García JC, Pérez-Padilla JR. Manual para el uso y la interpretación de la espirometría por el Médico. Asociación Latinoamericana del Tórax (ALAT), 2007. Fecha de consulta: junio 26, 2012. Disponible en: http:// www.alatorax.org.

17. Vázquez-García JC, Pérez-Padilla JR. Interpretación de la espirometría en 10 pasos. Guía de bolsillo. Asociación Latinoamericana del Tórax (ALAT), 2007. Fecha de consulta: junio 26, 2012. Disponible en: http://www. alatorax.org.

18. Benítez-Pérez RE, Torre-Bouscoulet L, Villca-Alá N, Del Río-Hidalgo RF, Pérez-Padilla R, Vázquez García JC, et al. Espirometría: Recomendaciones y procedimiento. Neumol Cir Torax 2016; 75:173-190.

19. Hankinson JL, Odencrantz JR, Fedan KB. Spirometric reference values from a sample of the general $U$. $S$. population. Am J Respir Crit Care Med 1999;159(1):179-187.

20. Pérez-Padilla JR, Regalado-Pineda J, Vázquez JC. Reproducibilidad de espirometrías en trabajadores mexicanos y valores de referencia internacionales. Salud Pub Mex 2001;43(2):113-121.

21. Pérez-Padilla R, Torre-Bouscoulet L, Vázquez-García JC, et al.; Grupo PLATINO. Spirometry reference values after inhalation of 200 microg of salbutamol. Arch Bronconeumol 2007;43(10):530-534.
22. Crapo RO, Jensen RL, Wanger JS. Single breath carbon monoxide difussing capacity. Clin Chest Med 2001;22(4):637-650.

23. American Thoracic Society. Single breath carbon monoxide difussing capacity. (Transfer factor). Recommendations for a standard technique-1995 update. Am J Respir Cri Care Med 1995;152(6 Pt 1):2185-2198.

24. Macintyre N, Crapo RO, Viegy G, et al. Standardization of the single breath determination of carbon monoxide uptake of the lung. Eur Respir J 2005;26(4):720-735.

25. Graham BL, Brusasco V, Burgos F, Cooper BG, Jensen $R$, Kendrick A, Maclntyre NR, Thompson BR, Wanger J. 2017 ERS/ATS standards for single-breath carbon monoxide uptake in the lung. Eur Respir J. 2017; 3:1-31.

26. Vázquez-García JC, Gochicoa-Rangel L, Del Río-Hidalgo RF, Cid-Juárez S, Silva-Cerón M, Miguel-Reyes JL, et al. Prueba de difusión pulmonar de monóxido de carbono con técnica de una sola respiración (DL-COsb). Recomendaciones y procedimiento. Neumol Cir Torax, 2016; 75:161-172.

27. Vázquez-García JC, Pérez-Padilla R, Casas A, SchönffeldtGuerrero P, Pereira J, Vargas-Domínguez C, et al. Reference Values for the Diffusing Capacity Determined by the Single-Breath Technique at Different Altitudes: The Latin American Single-Breath Diffusing Capacity Reference Project. Respir Care. 2016; 61:1217-1223.

28. Weisman IM, Zeballos RJ. Clinical exercise testing. Clin Chest Med 2001;22(4):679-702,viii.

29. American Thoracic Society; American Collage of Chest Physicians. ATS/ACCP statement on cardiopulmonary exercise testing. Am J Respir Cri Care Med 2003;167(2):211-277.

30. Evaluation of impairment/disability secondary to respiratory disorders. American Thoracic Society. Am Rev Respir Dis 1986;133(6):1205-1209.

31. Guidelines for the evaluation of impairment/disability in patients with asthma. American Thoracic Society. Medical Section of the American Lung Association. Am J Respir Cri Care Med 1993;147(4):1056-1061.

32. Cotes JE. Rating respiratory disability: a report on behalf of a working group of group of the European Society for Clinical Respiratory Physiology. Eur Respir J 1990;3(9):1074-1077.

33. Cochiarella L, Anderson GBJ. Guides to the evaluation of permanent impairment. The respiratory system. 5th edition. Chicago: AMA Press; 2000.

\author{
$\triangle$ Correspondencia: \\ Dr. Juan Carlos Vázquez-García \\ Dirección de Enseñanza, Instituto Nacional de \\ Enfermedades Respiratorias \\ Ismael Cosío Villegas. \\ Calzada de Tlalpan Núm. 4502, \\ Sección XVI, 14080, \\ Del. Tlalpan, CDMX. \\ Tel/Fax: 5554871744 \\ Correo electrónico: drjcvazquez@gmail.com
}

Los autores declaran no tener conflicto de intereses. 